\title{
Evaluation of Analysis Techniques for Fluted-Core Sandwich Cylinders
}

\author{
Andrew E. Lovejoy ${ }^{1}$ and Marc R. Schultz ${ }^{2}$ \\ NASA Langley Research Center, Hampton, VA 23681
}

\begin{abstract}
Buckling-critical launch-vehicle structures require structural concepts that have high bending stiffness and low mass. Fluted-core, also known as truss-core, sandwich construction is one such concept. In an effort to identify an analysis method appropriate for the preliminary design of fluted-core cylinders, the current paper presents and compares results from several analysis techniques applied to a specific composite fluted-core test article. The analysis techniques are evaluated in terms of their ease of use and for their appropriateness at certain stages throughout a design analysis cycle (DAC). Current analysis techniques that provide accurate determination of the global buckling load are not readily applicable early in the DAC, such as during preliminary design, because they are too costly to run. An analytical approach that neglects transverse-shear deformation is easily applied during preliminary design, but the lack of transverse-shear deformation results in global buckling load predictions that are significantly higher than those from more detailed analysis methods. The current state of the art is either too complex to be applied for preliminary design, or is incapable of the accuracy required to determine global buckling loads for fluted-core cylinders. Therefore, it is necessary to develop an analytical method for calculating global buckling loads of fluted-core cylinders that includes transverse-shear deformations, and that can be easily incorporated in preliminary design.
\end{abstract}

\section{Introduction}

$\mathrm{I}_{\mathrm{te}}^{\mathrm{n}}$ an effort to reduce the weight of launch vehicles, numerous materials and structurally efficient construction techniques have been investigated. Because global buckling is typically a driving factor in cylinder design for launch vehicles, structural concepts that have high bending stiffness and low mass have been developed. One such concept is sandwich construction, which consists of a lightweight core between two stiff faces. In sandwich construction, the faces carry the majority of the in-plane load, and the core carries the transverse-shear load and keeps the faces separated. Typical sandwich construction, shown in Fig. 1a, has a core such as foam, balsa wood, or honeycomb that has stiffness properties that are of the same order of magnitude in the two in-plane directions. However, one advantage of sandwich construction is the ability to produce directional cores using various materials and construction methods. Different types of fluted-core directional sandwich core constructions are shown in Fig. $1 \mathrm{~b}-\mathrm{f}$. Analysis techniques for fluted-core sandwich construction are evaluated in this paper in an effort to identify a method that is appropriate for analyzing fluted-core cylinders during the preliminary design portion of a design analysis cycle (DAC). Preliminary design capability of fluted-core cylinders is very important so that this structural concept can be included in early design studies for future launch vehicle components, such as for the Space Launch System (SLS) currently under development at NASA.

Fluted-core sandwich concepts have been explored for structures representative of both aircraft and launchvehicle structures, ${ }^{1-5}$ and consist of face sheets and a web core arranged in different ways, as shown in Fig. 1b-f. Moreover, the cells in a fluted core can be filled ${ }^{1}$ or unfilled. ${ }^{2-5}$ The present authors consider corrugated-core sandwich construction (Fig. 1b) to be a subset of fluted-core sandwich construction, however, in many references the terminology has been used interchangeably. For example, in Refs. 6 and 7, "truss" and "fluted" were respectively used for what the present authors would refer to as corrugated-core sandwich. Herein, corrugated core is distinguished from other fluted core as follows: corrugated-core sandwich construction contains faces in which every other segment is thicker than the adjacent segments due to the added thickness from the sinuous plies that

\footnotetext{
${ }^{1}$ Research Aerospace Engineer, Structural Mechanics \& Concepts Branch, MS 190, Senior Member AIAA, Andrew.E.Lovejoy@nasa.gov.

${ }^{2}$ Research Aerospace Engineer, Structural Mechanics \& Concepts Branch, MS 190, Senior Member AIAA.
} 
form the web, while the other fluted-core sandwich constructions contain faces in which all segments have identical thickness and lay-up. It should be noted, however, that the complications associated with the analysis of corrugatedcore sandwich construction and other fluted-core sandwich constructions are essentially the same. The remainder of this paper focuses on a specific fluted-core sandwich cylinder design (Section II), presents and compares several analysis techniques (Sections IIIA - IIIB), evaluates the merits of these techniques in the design of fluted-core sandwich cylinders (Section IV), and lastly, outlines future work that is needed to facilitate the analysis of flutedcore sandwich construction in preliminary design (Section V).

\section{Fluted-core Sandwich Cylinder Definition}

The fluted-core sandwich geometry considered in this paper is shown in Fig. 2, with the geometric portions of the fluted-core sandwich identified; the segments are considered to be made of IM7/8552 carbon/epoxy composite. ${ }^{8}$ Many methods exist for manufacturing a fluted-core sandwich. The method considered herein is consistent with those presented in Refs. $2-4$. That is, the assumed construction method uses composite trapezoidal tubes to form the fluted core, upon which are adhered composite faces. The core tubes have radii at the four corners that create a region that is encapsulated by core wall and face. In practice, these regions are not left empty, so herein the encapsulated region is considered to be filled with unidirectional composite material, creating a noodle as identified in Fig. 2. This construction concept produces face segments that comprise face plies and one core-tube wall, and core-web segments (hereafter referred to simply as web segments) that comprise two core-tube walls. Herein, the phrase fluted-core segments refers to all of the face and web segments. The noodle region is bounded on one side by face plies, and on the other two sides by core tube wall. The considered construction used two-ply [-45/45] core tubes and six-ply [0/90/90/0/-45/45] additional faces. When combined, the face segments have an eight-ply quasiisotropic [45/-45/0/90/90/0/-45/45] stacking sequence, and the web segments have a four-ply unsymmetric [-45/45/45/45] stacking sequence. Due to the manner in which the fluted core is constructed, the normal directions of the webs are oriented in essentially the same circumferential direction, as shown by the normal vectors, $\mathrm{n}$, depicted in Fig. 2.

All of the relevant cross-sectional dimensions for the considered cylindrical test article are shown in Fig. 2. The cylinder is considered to be 120-in. long, and has an outer-mold-line (OML) radius of 78 in. The internal height of the fluted-core tubes is 0.65 in., and the angle between the faces and the web is 60 degrees. With the exception of the curvature, these are essentially the same dimensions as the subscale test articles discussed in Ref. 3 and the curved panel of Ref. 4., and the cylinder described herein is representative of the test article shown in Fig. 3, which was manufactured by The Boeing Company. In order to prevent premature failures within the bending boundary layer of the test article, the ends of the cylinder have 9.5-in.-long doublers on both the inside and outside faces. The doublers have the same eight-ply lay-up as the skin for the endmost $6.5 \mathrm{in}$., and drop 2 plies over each of the next three inches, starting with the innermost 0/90, followed by the remaining 0/90, the innermost $-45 / 45$ plies, and finally the outermost $-45 / 45$ plies.

\section{Analysis Methods}

Typically, the degree of detail included in an analysis is dependent upon the stage of the DAC in which the analysis is being performed. Analyses performed earlier in the DAC, such as during preliminary design, are required to be simple to generate and quick to perform, and are intended to provide a gross comparative measure by which to rank potential designs. Analyses performed during the middle of the DAC require more detail and are used to generate designs from which the final design will be down-selected. The most detailed analyses occur during detailed design, and are performed to evaluate and verify that all design requirements are met by the final design. The detailed analyses used in the middle and end of the DAC are usually much more complex to generate and can be very time consuming to perform. Additionally, multiple analyses may be carried out using various models and methods to complete these detailed analyses. In this paper, three fluted-core-cylinder analysis methods are considered: 1) an analytical solution developed by the authors, 2) the method of Ref. 2 using the optimization code GENOPT $^{9}$ coupled with the structural-analysis code BIGBOSOR $4,{ }^{10,11}$ and 3 ) finite-element analysis. These analysis methods represent the current state of the art pertaining to composite fluted-core structural analysis, and are described in more detail in subsequent sections. The suitability of these three analysis methods for fluted-core sandwich structures is evaluated, and recommendations are made about their appropriateness for use within the DAC. Of particular interest is their capability to accurately predict the global buckling load, and their suitability for the preliminary design portion of the DAC. 


\section{A. Analytical Solution}

An analytical solution for the global buckling of fluted-core sandwich cylinders was developed in the interest of providing a quick analysis method for use in preliminary design of fluted-core cylinders. A preliminary survey of the literature has found that transverse-shear deformation has been incorporated in fluted-core/corrugated construction by numerous researchers. ${ }^{12-22}$ However, these efforts have been applied primarily to constructions utilizing isotropic materials. ${ }^{12-20}$ Additionally, the references use analytical methods coupled with design curves or finite-element analysis to determine equivalent properties that are then incorporated into a smeared finite-element analysis. While these references provide insight for incorporating transverse shear in the composite fluted-core analysis, a stand-alone method without the cumbersome design curves or analysis methods is preferable. In this paper, a global buckling analytical approach that does not incorporate transverse-shear deformation is developed and investigated. The global buckling analytical approach is then coupled with other analytical solutions, such as those applicable to local buckling of the segments and strength analysis of the segments and noodles, to evaluate the general response of the cylinder. The doublers at the cylinder ends were not considered in the analytical solution developed herein.

The global buckling analysis approach developed herein considers the cross-section reduced to a shell with the stiffness properties averaged or smeared over the shell; this follows the approach described in NASA SP-8007, ${ }^{23}$ and is based on the work of Jones. ${ }^{24}$ Development of the analytical solution for fluted-core cylinder global buckling is presented in Appendix A. For uniaxial compression load, where the $x$-axis is aligned with the cylinder axis, the critical buckling running load of the cylinder is computed using the buckling equation:

$$
\bar{N}_{x}=\left(\frac{L}{m \pi}\right)^{2} \frac{\left|\begin{array}{lll}
A_{11}^{*} & A_{12}^{*} & A_{13}^{*} \\
A_{12}^{*} & A_{22}^{*} & A_{23}^{*} \\
A_{13}^{*} & A_{23}^{*} & A_{33}^{*}
\end{array}\right|}{\left|\begin{array}{ll}
A_{11}^{*} & A_{12}^{*} \\
A_{12}^{*} & A_{22}^{*}
\end{array}\right|}
$$

where $\bar{N}_{x}$ is the average running load, $L$ is the cylinder length, and the $A^{*}$ terms are given in the appendix and are stiffness terms that are functions of cylinder geometry, material, and $m$ and $n$. The $m$ and $n$ are the number of half waves in the axial direction and number of full waves in the circumferential direction, respectively, for the cylinder buckling modes. The values of $m$ and $n$ that produce the lowest value of $\bar{N}_{x}$ are determined, and the resulting value is the critical buckling running load, $\bar{N}_{x}^{c r}$. For this paper, the buckling equation was implemented in a spreadsheet to determine the critical buckling load as a function of the composite fluted-core-cylinder design parameters by considering all combinations of $m$ and $n$ up to a value of 20. The equation is easily implemented in any number of ways, but the spreadsheet approach was chosen due to the automatic calculation of design loads when design variables were changed. Also included in the spreadsheet were standard analytical solutions for local buckling of the fluted-core segments, and strength analysis of the fluted-core segments and noodles. Local buckling is calculated for each segment by assuming it is a simply-supported plate and using a classical buckling equation. ${ }^{25}$ The length of the plate for local buckling is equal to the cylinder length, and the plate width is equal to the adjusted length of the segment for which local buckling is being calculated. The adjusted length of the segment is the unsupported portion of the segment that is between the noodles. The critical local-buckling $\bar{N}_{x}$ is calculated by considering the appropriate load distribution to the segments and noodles according to the axial-stiffness weighted cross section of each portion. Separate analyses (based on classical lamination theory ${ }^{26}$ and the maximum stress failure criterion, using the material information of Ref. 8) were performed for each of the segment laminates to determine the strain at which first ply failure would occur for each laminate, and this value was used as a laminate failure strain with which to calculate material-failure loads; the noodle was considered to act as the unidirectional material for material failure prediction. The running load that resulted in material failure was calculated using the stiffness of each segment and the discussed strain allowable. Including the global buckling, local buckling, and strength calculations provides a set of analytical solutions that is representative of the types of responses that would be included at the preliminary design stage. To evaluate the analytical method, and in particular the global buckling solution for the fluted-core construction, it was first applied to the cylinder presented in Section II. The analytical calculated buckling load for this cylinder is 3,715 kips, and will be compared to the solutions obtained by the methods presented in Sections IIIB 
and IIIC. The local buckling and material-failure loads for the cylinder are predicted to be 5,631 and 3,035 kips, respectively.

\section{B. GENOPT/BIGBOSOR4}

Analysis was performed by Bushnell and Rankin ${ }^{2}$ on the fluted-core cylinder, presented in Section II, using GENOPT and the shell-of-revolution analysis code BIGBOSOR4. GENOPT is a program that is used to link analysis methods within a program system with which a user can perform analysis or optimization. In this case, BIGBOSOR4 was linked to provide the required design analyses. In their approach, the cylinder was approximated as a portion of a huge torus such that the fluted-core runs around the large circumference of the torus. This means that the axial coordinate of the cylinder becomes the circumferential coordinate of the torus, and the circumferential coordinate of the cylinder becomes the meridional coordinate of the torus. As long as the torus is large enough, the response of a small segment of the torus will approximate that of a cylinder provided that: 1) the ends of the cylinder are simply-supported, 2) the pre-buckled state is a membrane state (no pre-buckling bending), and 3) the torus circumferential radius is much larger than the torus meridional radius. ${ }^{2}$

Ref. 2 uses very detailed representation of all portions of the fluted-core construction, including the face segments, web segments, and the sublaminate segments that enclose the noodle region. However, the doublers at the cylinder ends were not considered their model. The global buckling load for the cylinder is reported in Ref. 2 as 2,513 kips. In the reference, several methods were utilized to model the noodle region. Based on the local model that Bushnell and Rankin report as being the most appropriate modeling approach, ${ }^{2}$ the predicted local buckling load is 5,977 kips, and the predicted material-failure load is 2,536 kips. $^{2}$

Unfortunately, GENOPT and BIGBOSOR4 are specialized software codes that require an intimate knowledge of their use, particularly when setting up the process for a new structural construction. Therefore, very few analysts are capable of using this method to develop the necessary detailed models without a significant learning curve. Additionally, the approach is not particularly suitable for preliminary design where many designs will be investigated because a new analysis process will have to be developed for each design concept. ${ }^{2}$

\section{Finite-Element Analysis}

There are many finite-element models of varying degrees of complexity that can be developed to model the fluted-core construction. For comparison with test results of small (11 in. by $5.75 \mathrm{in}$. and smaller), flat test articles, Schultz, et al. ${ }^{3}$ developed very detailed models to simultaneously capture numerous failure modes, such as local buckling and high stresses, seen with the fluted-core construction. Their model used shell elements to represent the skin and flute portions, and solid elements to represent the noodle region. The advantage of this modeling approach for simultaneously capturing numerous failure modes is quickly offset as the size of the structure being analyzed increases. As such, this modeling method is better suited for detailed analysis of small structures, or for detailed local analysis of larger structures. It was this computational cost that led Oremont and Schultz ${ }^{4}$ to develop a more efficient approach for modeling the fluted-core construction for larger test specimens. Their simplified approach replaces the solid elements of the noodle region with a set of shell elements in a so-called shell-noodle approach. This change in element type reduces the size of the model, but at the same time, the ability to accurately recover stresses and strains in the noodle region is diminished. Therefore, to more accurately determine noodle-region strains and stresses, a solid-noodle model can be embedded within a larger shell-noodle model, or can be analyzed independently as a local model with global-model boundary conditions applied. Additionally, the solid-noodle model is required to tune the shell-noodle model by determining the correct property sets that will most accurately represent the noodle region. This global and local approach is most applicable to very detailed analysis of a completed design. Thus, the modeling approaches of Refs. 3 and 4 are not readily applicable early in the design process.

In this paper, an even more simplified finite-element approach was used to analyze the considered cylinder. Global and local models were developed with the inner and outer face sheets and the core webs modeled using shell finite elements. A single element was used to span the width of each face and core web segment in the complete cylinder model (Fig. 4). Beam finite elements were included along the face and web intersections to represent the noodles. Simply-supported boundary conditions were applied to the ends of each segment. A local model that was the length of the cylinder was then extracted from the cylinder model, and consisted of seven full and two half flutes. The mesh of this local model was significantly refined to evaluate local buckling and strains, and is shown in Fig. 5. The long and short face segments had 14 and 6 elements along the cross section, respectively, and the webs had 8 elements along the cross section. Simply-supported boundary conditions were applied to the ends of the local model, and symmetry conditions were applied to the face edges along the entire length of the local model. A uniform end displacement was applied to both the global and the local models, and the constraint forces were 
summed to determine the applied load. A complete analysis was performed on both the global and local models using the general-purpose finite-element code MSC/NASTRAN ${ }^{27}$ using QUAD4 and BAR elements. The doublers at the cylinder ends were considered in both the global and local finite-element models.

A linear bifurcation buckling analysis was performed using the model shown in Fig. 4 to determine the global buckling load. The predicted cylinder global buckling load is 2,535 kips, which corresponds to the mode shown in Fig. 6. Linear bifurcation analysis was also performed on the local model of Fig. 5 to determine local buckling of the face and core web segments. The predicted local buckling load is 3,371 kips, and buckling occurs in the wider face segments as shown in Fig. 7. Nonlinear static analysis was carried out using the local model to determine materialfailure load. The Hill failure criterion as implemented in MSC/NASTRAN was applied to the local model subjected to the global buckling load, and failure indices were calculated. The failure index is the interactive failure theory equation that incorporates the calculated stresses and stress allowables such that a value greater than or equal to 1.0 indicates failure. Applying the global buckling load leads to the maximum calculated failure index for the cylinder being 0.7114, which occurs in the face segments adjacent to the doublers as shown in Fig. 8. Failure indices in the doubler region of the faces are not plotted because all failure indices in this region are less than 0.6. The calculated maximum failure index indicates that material failure is predicted to occur at a total load of 3,004 kips.

\section{Comparison and Discussion}

The analysis results from the three considered analysis methods for the fluted-core cylinder that was defined in Section II are summarized in Table 1. Comparison between the finite-element results and the GENOPT/BIGBOSOR4 results for global buckling of the fluted-core sandwich cylinder reveals extremely good agreement. In contrast, the global buckling load predicted from the analytical solution is higher than that predicted by the other two methods by $47 \%$. These global buckling results are discussed in more detail below.

The analytical and GENOPT/BIGBOSOR4 local buckling loads show agreement within $6 \%$, but are about $122 \%$ and $135 \%$ higher than the finite-element solution, respectively. The analytical local buckling and the GENOPT/BIGBOSOR4 local buckling loads are higher because the fluted-core segments for local buckling are shorter than those in the finite-element model because the unsupported length of the segments are used as the buckling lengths. In the finite-element model, the noodles are represented by beams at the shell intersections with no explicit noodle region so the local buckling lengths of face and web segments extend to the intersection location of the faces and webs. For example, the long face segment in the analytical solution and the GENOPT/BIGBOSOR4 model is approximately 1.0 in., and the long face segment in the finite-element models is approximately 1.3 inches. The increased face segment length in the finite-element analysis results in lower, more conservative local buckling loads.

The material-failure loads from the analytical solution and the finite-element method agree within $1 \%$. Again, this good agreement is an expected result since these two methods model only the full-segment laminates, and ignore the reduced-thickness portions of the faces and webs that encapsulate the noodle. The material-failure load from Ref. 2, however, is about $20 \%$ lower than those from the analytical solution and the finite-element method. Ref. 2 does not indicate the location of the critical material failure, and since Ref. 2 does include details such as the sublaminate enclosing the noodle and the noodle itself, it is possible that the location of the critical material failure is associated with a stress concentration in the vicinity of the noodle, which could account for the lower load reported in the reference.

Due to the large discrepancy between the global buckling loads predicted by the analytical solution and the other two methods, additional finite-element analyses using the finite-element codes NASTRAN and Abaqus ${ }^{28}$ were performed. Reference 2 reported good agreement between the method presented therein and the finite-element code STAGS ${ }^{29}$ which also agree well with the NASTRAN results presented herein, so the finite-element code Abaqus was used in the current study to provide an additional solution. To simplify the translation of the global model of Fig. 4, and to avoid differences that may arise due to different beam formulations in the codes, the noodles were removed from the design in these additional analyses. The resulting models are noodle-less, all-shell finite-element models of a cylinder having the same face and web segments as defined in Section II, and with QUAD4 elements in the NASTRAN model and S4 elements in the Abaqus model. The critical global buckling loads calculated with the analytical solution, NASTRAN, and Abaqus for this noodle-less composite cylinder are given in Table 2, and the corresponding NASTRAN and Abaqus mode shapes are shown in Fig. 9. While the modes shown in Fig. 9 compare favorably, the buckling loads in Table 2 do not. The Abaqus and the analytical global buckling loads are within 3\% of each other. However, the NASTRAN global-buckling load is approximately $38 \%$ below the analytical solution value. To address the differences between the NASTRAN and Abaqus results, the mesh was refined to determine if the finite-element solutions were converged. The refined mesh used two elements to span the lengths of the fluted- 
core segments, and the buckling loads for the refined mesh are shown in Table 2. The predicted critical buckling load from the refined NASTRAN model is only $2 \%$ lower than the original NASTRAN prediction, but the predicted buckling load from the refined Abaqus model is 33\% lower than the original Abaqus prediction. The predicted critical buckling loads from the refined NASTRAN and Abaqus models are within $8 \%$. Therefore, NASTRAN appears to provide a converged solution with only one element per segment, but Abaqus requires at least two elements per segment. The original Abaqus model acts overly stiff in transverse-shear, and it is speculated that good agreement is seen between the coarse Abaqus model and the analytical model because transverse-shear deformation is ignored in the analytical global buckling method. Another finite-element study to explore this transverse-shear issue is described next.

The transverse-shear response that was examined with additional analyses considered the relative shearing motion of the two faces, and is a separate issue from whether transverse shear is important within the individual face or web segments themselves. The axial (along the webs) transverse-shear response is governed by the in-plane shear of the webs, and the circumferential (perpendicular to the webs) transverse-shear response is dominated by the bending response of the face and web segments. To examine the relative shearing stiffness between the finiteelement codes, a study using several small, flat fluted-core models was conducted with several combinations of elements spanning the lengths of the segments. Meshes are designated by the number of elements spanning the face and web segments, respectively. Thus, a mesh designated as $3 / 4$ has three elements that span the face segments and four elements that span the web segments. Two meshes were considered for the axial transverse-shear analyses: 1/1 and $2 / 2$ meshes. Seven meshes (up to a 3/4 mesh) were considered for the circumferential transverse-shear analyses. Figure 10 shows the 1/1 and 3/4 meshes, where it is seen that the two face sheets are not identical in width due to the trapezoidal flute construction; thus there is a wide face and a narrow face. To examine the relative shear stiffness of the various models in the three finite-element codes, one edge of the wide face was restrained from movement, and the narrow face was loaded with a uniform traction of $0.5 \mathrm{lbs} / \mathrm{in}$. along all ten face-and-web-segment interface lines in either the axial or circumferential direction, as shown in Fig. 10. Additionally, the wide face sheet had all the nodes at the face-and-web-segment intersections restrained from transverse displacement. The axial and circumferential transverse-shear displacements obtained from the three finite-element codes are shown in Tables 3 and 4, respectively. The presented displacement is the average displacement of the narrow face edge that is opposite the wide face restrained edge, as indicated in Figure 10. It is seen that for the axial transverse-shear displacement, all three finite-element codes give very comparable results; the predicted displacements are within $7 \%$ for the $1 / 1$ mesh and within $3 \%$ for the $2 / 2$ mesh. The results of Table 3 demonstrate that the $1 / 1$ mesh gives results that are fairly converged for the axial transverse-shear stiffness. However, for the displacements under circumferential transverseshear loading shown in Table 4, the Abaqus-predicted displacement is over $80 \%$ lower than the NASTRANpredicted displacement for the $1 / 1$ mesh. Additionally, it is seen in the table that refinement to the $3 / 4$ mesh is needed for the Abaqus-predicted displacement to be within 7\% of NASTRAN. The transverse-shear study that was conducted, in conjunction with the global buckling results, indicates that transverse shear is very important to the buckling response of fluted-core structure, and in particular, the circumferential transverse shear plays an important role. Magnified NASTRAN deformations for the circumferential transverse strain models are shown in Fig. 11 for the $1 / 1,2 / 2$, and $3 / 4$ meshes. The bending of the fluted-core segments that results in the effective low circumferential transverse-shear stiffness is seen in this figure. It will be necessary to include transverse-shear deformation in an analytical global buckling solution for fluted-core sandwich structures to ensure that the buckling loads obtained are meaningful.

\section{Conclusions}

Three analysis methods for a composite fluted-core cylinder, namely an analytical approach, GENOPT coupled with BIGBOSOR4, and finite-element analysis, were compared to determine their appropriateness for various portions of the design analysis cycle (DAC), particularly for preliminary design. The analytical method is easily implemented in a preliminary design study. However, the analytical method developed herein does not include transverse-shear deformation, which leads to significant overestimation of the global buckling load, therefore, is not recommended for use in analyzing fluted-core cylinders. The GENOPT/BIGBOSOR4 approach can give analysis results similar to the detailed finite-element approach, so it is also ideal later in the design process. However, the optimization nature of the GENOPT/BIGBOSOR4 method means that it can be used to develop design sizing. However, as previously mentioned, the approach requires specialized code knowledge, and is not particularly suitable for preliminary design because a new analysis process will have to be developed for each design concept. Finite-element analysis is a common structural analysis method. Therefore, most structural analysts will be able to use it to analyze fluted-core structure. However, it has been shown that detailed analysis of the fluted-core can 
require very complex models; ${ }^{3,4}$ this suggests finite-element analysis is generally well suited for the final detailed design phase of the DAC, where a final design is being analyzed to ensure it satisfies the requirements. It was demonstrated that relatively simple finite-element models can be used to analyze the fluted-core for basic response, such as global and local buckling, and overall strain requirements. This more simplified finite-element approach could be used earlier in the DAC, and if coupled with a script to automatically generate the finite-element models might be usable in an early design study. However, even this simplified finite-element approach is still more modeling-and-analysis intensive than is desirable for preliminary design.

It was shown that it is important to accurately predict the transverse-shear response of the overall fluted-core sandwich construction because transverse-shear effects can have a significant influence on the global buckling load prediction. Therefore, to better facilitate preliminary design of fluted-core construction, the analysis method should include transverse-shear deformation, particularly in the circumferential direction. Developing an analytical method for calculating the global buckling load for composite fluted-core cylinders, similar to the one developed herein that is easily applied during preliminary design, but one that incorporates transverse shear, is important for anyone wishing to consider a flute-core construction in the design of future launch-vehicle components.

\section{Appendix A: Analytical Solution Equation Development}

Development of the buckling equations for the fluted-core cylinder begins by modifying the equations of Jones ${ }^{13}$ so that we can use the familiar A, B, and D matrix stiffness terms for laminates. ${ }^{25,26}$ The general form for the stability criterion, where the cylinder axis is aligned with the $\mathrm{x}$-axis, is given by

$$
\bar{N}_{x}\left(\frac{m \pi}{L}\right)^{2}+\bar{N}_{y}\left(\frac{n}{R}\right)^{2}=A_{33}^{*}+A_{23}^{*}\left(\frac{A_{13}^{*} A_{12}^{*}-A_{11}^{*} A_{23}^{*}}{A_{11}^{*} A_{22}^{*}-A_{12}^{* 2}}\right)+A_{13}^{*}\left(\frac{A_{12}^{*} A_{23}^{*}-A_{13}^{*} A_{23}^{*}}{A_{11}^{*} A_{22}^{*}-A_{12}^{* 2}}\right)
$$

where

$L=$ cylinder length

$R=$ cylinder radius (to reference surface)

$m=$ number of half waves in axial direction

$n=$ number of full waves in circumferential direction

$\bar{N}_{x}=$ running load in axial direction

$\bar{N}_{y}=$ running load in circumferential direction

$A_{i j}^{*}=$ stiffness terms related to laminate $\mathrm{A}, \mathrm{B}$, and D matrix stiffness terms and stiffener properties, to be defined subsequently by Eq. (A4)

For axial compression loading only, Eq. (A1) reduces to

$$
\bar{N}_{x}=\left(\frac{L}{m \pi}\right)^{2} \frac{\left|\begin{array}{lll}
A_{11}^{*} & A_{12}^{*} & A_{13}^{*} \\
A_{12}^{*} & A_{22}^{*} & A_{23}^{*} \\
A_{13}^{*} & A_{23}^{*} & A_{33}^{*}
\end{array}\right|}{\left|\begin{array}{ll}
A_{11}^{*} & A_{12}^{*} \\
A_{12}^{*} & A_{22}^{*}
\end{array}\right|}
$$

The constitutive equations, corresponding to the plane stress condition, and ignoring thermal and hygrothermal effects, are 


$$
\left\{\begin{array}{l}
N_{x} \\
N_{y} \\
N_{x y} \\
M_{x} \\
M_{y} \\
M_{x y}
\end{array}\right\}=\left[\begin{array}{llllll}
A_{11} & A_{12} & A_{16} & B_{11} & B_{12} & B_{16} \\
A_{12} & A_{22} & A_{26} & B_{12} & B_{22} & B_{26} \\
A_{16} & A_{26} & A_{66} & B_{16} & B_{26} & B_{66} \\
B_{11} & B_{12} & B_{16} & D_{11} & D_{12} & D_{16} \\
B_{12} & B_{22} & B_{26} & D_{12} & D_{22} & D_{26} \\
B_{16} & B_{26} & B_{66} & D_{16} & D_{26} & D_{66}
\end{array}\right]\left\{\begin{array}{c}
\varepsilon_{x} \\
\varepsilon_{y} \\
\gamma_{x y} \\
\kappa_{x} \\
\kappa_{y} \\
\kappa_{x y}
\end{array}\right\}
$$

where the $A_{i j}, B_{i j}$ and $D_{i j}$ terms are the familiar laminate stiffness terms. ${ }^{25,26}$

The $A_{i j}^{*}$ stiffness terms for a laminated cylinder with stringer and ring stiffeners, which serve as the basis for the current method, are given in a manner consistent with the notation of NASA SP- $8007^{23}$ as

$$
\begin{gathered}
A_{11}^{*}=\bar{E}_{x}\left(\frac{m \pi}{L}\right)^{2}+\bar{G}_{x y}\left(\frac{n}{R}\right)^{2} \\
A_{12}^{*}=\left(\bar{E}_{x y}+\bar{G}_{x y}\right)\left(\frac{m \pi}{L}\right)\left(\frac{n}{R}\right) \\
A_{13}^{*}=\left(\frac{\bar{E}_{x y}}{R}\right)\left(\frac{m \pi}{L}\right)+\bar{C}_{x}\left(\frac{m \pi}{L}\right)^{3}+\left(\bar{C}_{x y}+2 \bar{K}_{x y}\right)\left(\frac{m \pi}{L}\right)\left(\frac{n}{R}\right)^{2} \\
A_{22}^{*}=\bar{G}_{x y}\left(\frac{m \pi}{L}\right)^{2}+\bar{E}_{y}\left(\frac{n}{R}\right)^{2} \\
A_{23}^{*}=\left(\bar{C}_{x y}+2 \bar{K}_{x y}\right)\left(\frac{m \pi}{L}\right)^{2}\left(\frac{n}{R}\right)+\left(\frac{1}{R}\right) \bar{E}_{y}\left(\frac{n}{R}\right)+\bar{C}_{y}\left(\frac{n}{R}\right)^{3} \\
A_{33}^{*}=\bar{D}_{x}\left(\frac{\mathrm{m} \pi}{\mathrm{L}}\right)^{4}+\bar{D}_{x y}\left(\frac{\mathrm{m} \pi}{\mathrm{L}}\right)^{2}\left(\frac{n}{R}\right)^{2}+\bar{D}_{y}\left(\frac{n}{R}\right)^{4}+\left(\frac{2}{R}\right) \bar{C}_{x y}\left(\frac{\mathrm{m} \pi}{\mathrm{L}}\right)^{2}+\left(\frac{2}{R}\right) \bar{C}_{y}\left(\frac{n}{R}\right)^{2}+\left(\frac{1}{R^{2}}\right) \bar{E}_{y}
\end{gathered}
$$

where

$$
\begin{gathered}
\bar{E}_{x}=A_{11}+\frac{E_{s} A_{s}}{b} \\
\bar{E}_{y}=A_{22}+\frac{E_{r} A_{r}}{a} \\
\bar{E}_{x y}=A_{12} \\
\bar{G}_{x y}=A_{66} \\
\bar{C}_{x}=B_{11}+\frac{\widetilde{z}_{s} E_{s} A_{s}}{b} \\
\bar{C}_{y}=B_{22}+\frac{\widetilde{z}_{r} E_{r} A_{r}}{a} \\
\bar{C}_{x y}=B_{12} \\
\bar{K}_{x y}=B_{66} \\
\bar{D}_{x}=D_{11}+\frac{E_{s} I_{s}}{b}+\frac{\widetilde{z}_{s}^{2} E_{s} A_{s}}{b} \\
\bar{D}_{y}=D_{22}+\frac{E_{r} I_{r}}{a}+\frac{\widetilde{z}_{r}^{2} E_{r} A_{r}}{a}
\end{gathered}
$$




$$
\bar{D}_{x y}=4 D_{66}+2 D_{12}+\frac{G_{s} J_{s}}{b}+\frac{G_{r} J_{r}}{a}
$$

and

$$
\begin{aligned}
& b=\text { stringer spacing } \\
& E_{s}=\text { stringer Young's modulus } \\
& G_{s}=\text { stringer shear modulus } \\
& A_{s}=\text { stringer area } \\
& I_{s}=\text { stringer moment of inertia } \\
& J_{s}=\text { stringer torsional constant } \\
& \widetilde{z}_{s}=\text { offset of stringer axis } \\
& a=\text { ring spacing } \\
& E_{r}=\text { ring Young's modulus } \\
& G_{r}=\text { ring shear modulus } \\
& A_{r}=\text { ring area } \\
& I_{r}=\text { ring moment of inertia } \\
& J_{r}=\text { ring torsional constant } \\
& \widetilde{z}_{r}=\text { offset of ring axis }
\end{aligned}
$$

Next, consider a fluted-core sandwich cylinder where the repeated unit of the configuration is shown in Fig. A1. The repeated unit consists of six segments and four noodle regions, and the noodle regions are approximated using isosceles triangles. The stacking sequences are assumed to be the same for segments 1 and 4 , for segments 2 and 3 , and for segments 5 and 6 . Furthermore, the stacking sequences for segments 1-4 are considered to be symmetric about the mid-plane of the fluted-core sandwich. Next, the local $x$-axis is considered to be parallel to the flutes, or equivalently, parallel to the cylinder axis. Therefore, the local $y$-axis is tangential to the circumferential direction. The stiffness parameters defined above are calculated for this repeated unit and are used to calculate the response of the overall cylinder. For ease of calculation, it is assumed that the repeated unit is flat, i.e., that all segments are straight and that the lengths of segments 1 and 4 are equal $\left(l_{1 \& 4}\right)$ and the lengths of segments 2 and 3 are equal $\left(l_{2 \& 3}\right)$. This assumption is reasonable when the total thickness of the fluted-core sandwich is small compared to the radius of the cylinder. The lengths of segments 5 and $6\left(l_{5 \& 6}\right)$ are equal regardless of curvature. Equivalent engineering properties $\left(E_{1}, E_{2}\right.$, etc.) based upon the $\mathrm{A}, \mathrm{B}$, and $\mathrm{D}$ matrix values for the laminated segments of the repeated unit are used. Using the stated simplifications, the stiffness contributions from segments 1 and 4 have the form

$$
\begin{gathered}
\left(\bar{E}_{x}\right)_{1 \& 4}=2\left(\frac{\left(E_{1}\right)_{1 \& 4}}{\left(1-v_{12} v_{21}\right)_{1 \& 4}}\right) t_{1 \& 4}\left(\frac{l_{1 \& 4}}{l_{1 \& 4}+l_{2 \& 3}}\right) \\
\left(\bar{E}_{y}\right)_{1 \& 4}=2\left(\frac{\left(E_{2}\right)_{1 \& 4}}{\left(1-v_{12} v_{21}\right)_{1 \& 4}}\right) t_{1 \& 4}\left(\frac{l_{1 \& 4}}{l_{1 \& 4}+l_{2 \& 3}}\right) \\
\left(\bar{E}_{x y}\right)_{1 \& 4}=2\left(\frac{\left(v_{21} E_{1}\right)_{1 \& 4}}{\left(1-v_{12} v_{21}\right)_{1 \& 4}}\right) t_{1 \& 4}\left(\frac{l_{1 \& 4}}{l_{1 \& 4}+l_{2 \& 3}}\right) \\
\left(\bar{G}_{x y}\right)_{1 \& 4}=2\left(G_{12}\right)_{1 \& 4} t_{1 \& 4}\left(\frac{l_{1 \& 4}}{l_{1 \& 4}+l_{2 \& 3}}\right) \\
\left(\bar{C}_{x}\right)_{1 \& 4}=0 \\
\left(\bar{C}_{y}\right)_{1 \& 4}=0 \\
\left(\bar{C}_{x y}\right)_{1 \& 4}=0 \\
\left(\bar{K}_{x y}\right)_{1 \& 4}=0
\end{gathered}
$$




$$
\begin{gathered}
\left(\bar{D}_{x}\right)_{1 \& 4}=\frac{2}{3}\left(\frac{\left(E_{1}\right)_{1 \& 4}}{\left(1-v_{12} v_{21}\right)_{1 \& 4}}\right)\left(t_{1 \& 4}^{3}+3 d_{1 \& 4} t_{1 \& 4}^{2}+3 d_{1 \& 4}^{2} t_{1 \& 4}\right)\left(\frac{l_{1 \& 4}}{l_{1 \& 4}+l_{2 \& 3}}\right) \\
\left(\bar{D}_{y}\right)_{1 \& 4}=\frac{2}{3}\left(\frac{\left(E_{2}\right)_{1 \& 4}}{\left(1-v_{12} v_{21}\right)_{1 \& 4}}\right)\left(t_{1 \& 4}^{3}+3 d_{1 \& 4} t_{1 \& 4}^{2}+3 d_{1 \& 4}^{2} t_{1 \& 4}\right)\left(\frac{l_{1 \& 4}}{l_{1 \& 4}+l_{2 \& 3}}\right) \\
\left(\bar{D}_{x y}\right)_{1 \& 4}=\frac{2}{3}\left(4\left(G_{12}\right)_{1 \& 4}+2 \frac{\left(v_{21} E_{1}\right)_{1 \& 4}}{\left(1-v_{12} v_{21}\right)_{1 \& 4}}\right)\left(t_{1 \& 4}^{3}+3 d_{1 \& 4} t_{1 \& 4}^{2}+3 d_{1 \& 4}^{2} t_{1 \& 4}\right)\left(\frac{l_{1 \& 4}}{l_{1 \& 4}+l_{2 \& 3}}\right)
\end{gathered}
$$

where the subscript notation $i \& j$ means that the quantity is associated with segments $i$ and $j$, and that the quantity has the same value in segment $i$ as it does in segment $j$. For example, the subscript $1 \& 4$ refers to the fact that the quantity is associated with, and identical, for both segments 1 and 4 . The terms $d$ and $t$ are the segment offset from the reference surface, taken as the mid-surface for this derivation, and the thickness of the segment, repectively. Similarly, for segments 2 and 3,

$$
\begin{aligned}
& \left(\bar{E}_{x}\right)_{2 \& 3}=2\left(\frac{\left(E_{1}\right)_{2 \& 3}}{\left(1-v_{12} v_{21}\right)_{2 \& 3}}\right) t_{2 \& 3}\left(\frac{l_{2 \& 3}}{l_{1 \& 4}+l_{2 \& 3}}\right) \\
& \left(\bar{E}_{y}\right)_{2 \& 3}=2\left(\frac{\left(E_{2}\right)_{2 \& 3}}{\left(1-v_{12} v_{21}\right)_{2 \& 3}}\right) t_{2 \& 3}\left(\frac{l_{2 \& 3}}{l_{1 \& 4}+l_{2 \& 3}}\right) \\
& \left(\bar{E}_{x y}\right)_{2 \& 3}=2\left(\frac{\left(v_{21} E_{1}\right)_{2 \& 3}}{\left(1-v_{12} v_{21}\right)_{2 \& 3}}\right) t_{2 \& 3}\left(\frac{l_{2 \& 3}}{l_{1 \& 4}+l_{2 \& 3}}\right) \\
& \left(\bar{G}_{x y}\right)_{2 \& 3}=2\left(G_{12}\right)_{2 \& 3} t_{2 \& 3}\left(\frac{l_{2 \& 3}}{l_{1 \& 4}+l_{2 \& 3}}\right) \\
& \left(\bar{C}_{x}\right)_{2 \& 3}=0 \\
& \left(\bar{C}_{y}\right)_{2 \& 3}=0 \\
& \left(\bar{C}_{x y}\right)_{2 \& 3}=0 \\
& \left(\bar{K}_{x y}\right)_{2 \& 3}=0 \\
& \left(\bar{D}_{x}\right)_{2 \& 3}=\frac{2}{3}\left(\frac{\left(E_{1}\right)_{2 \& 3}}{\left(1-v_{12} v_{21}\right)_{2 \& 3}}\right)\left(t_{2 \& 3}^{3}+3 d_{2 \& 3} t_{2 \& 3}^{2}+3 d_{2 \& 3}^{2} t_{2 \& 3}\right)\left(\frac{l_{2 \& 3}}{l_{1 \& 4}+l_{2 \& 3}}\right) \\
& \left(\bar{D}_{y}\right)_{2 \& 3}=\frac{2}{3}\left(\frac{\left(E_{2}\right)_{2 \& 3}}{\left(1-v_{12} v_{21}\right)_{2 \& 3}}\right)\left(t_{2 \& 3}^{3}+3 d_{2 \& 3} t_{2 \& 3}^{2}+3 d_{2 \& 3}^{2} t_{2 \& 3}\right)\left(\frac{l_{2 \& 3}}{l_{1 \& 4}+l_{2 \& 3}}\right) \\
& \left(\bar{D}_{x y}\right)_{2 \& 3}=\frac{2}{3}\left(4\left(G_{12}\right)_{2 \& 3}+2 \frac{\left(v_{21} E_{2}\right)_{2 \& 3}}{\left(1-v_{12} v_{21}\right)_{2 \& 3}}\right)\left(t_{2 \& 3}^{3}+3 d_{2 \& 3} t_{2 \& 3}^{2}+3 d_{2 \& 3}^{2} t_{2 \& 3}\right)\left(\frac{l_{2 \& 3}}{l_{1 \& 4}+l_{2 \& 3}}\right)
\end{aligned}
$$

For the sake of developing their stiffness contributions, segments 5 and 6 are treated as stiffeners. Again, it is assumed that equivalent engineering properties derived from the $\mathrm{A}, \mathrm{B}$, and $\mathrm{D}$ matrix values are provided for these laminated segments. The stiffness contributions from segments 5 and 6 are then

$$
\begin{gathered}
\left(\bar{E}_{x}\right)_{5 \& 6}=\frac{2\left(E_{1} A\right)_{5 \& 6}}{l_{1 \& 4}+l_{2 \& 3}} \\
\left(\bar{E}_{y}\right)_{5 \& 6}=0 \\
\left(\bar{E}_{x y}\right)_{5 \& 6}=0 \\
\left(\bar{G}_{x y}\right)_{5 \& 6}=0
\end{gathered}
$$




$$
\begin{gathered}
\left(\bar{C}_{x}\right)_{5 \& 6}=0 \\
\left(\bar{C}_{y}\right)_{5 \& 6}=0 \\
\left(\bar{C}_{x y}\right)_{5 \& 6}=0 \\
\left(\bar{K}_{x y}\right)_{5 \& 6}=0 \\
\left(\bar{D}_{x}\right)_{5 \& 6}=\frac{2\left(E_{1} I_{2}\right)_{5 \& 6}}{l_{1 \& 4}+l_{2 \& 3}} \\
\left(\bar{D}_{y}\right)_{5 \& 6}=\frac{2\left(E_{1} I_{1}\right)_{5 \& 6}}{l_{1 \& 4}+l_{2 \& 3}} \\
\left(\bar{D}_{x y}\right)_{5 \& 6}=\frac{2\left(G_{23} J\right)_{5 \& 6}}{l_{1 \& 4}+l_{2 \& 3}}
\end{gathered}
$$

where $A, I_{1}, I_{2}$ and $J$ are the rectangular cross-section properties of the segments 5 and 6 . In a similar manner, the noodles are treated as stiffeners. The noodle stiffness contributions are

$$
\begin{gathered}
\left(\bar{E}_{x}\right)_{\text {noodle }}=\frac{4\left(E_{1} A\right)_{\text {noodle }}}{l_{1 \& 4}+l_{2 \& 3}} \\
\left(\bar{E}_{y}\right)_{\text {noodle }}=0 \\
\left(\bar{E}_{x y}\right)_{\text {noodle }}=0 \\
\left(\bar{G}_{x y}\right)_{\text {noodle }}=0 \\
\left(\bar{C}_{x}\right)_{\text {noodle }}=0 \\
\left(\bar{C}_{y}\right)_{\text {noodle }}=0 \\
\left(\bar{C}_{x y}\right)_{\text {noodle }}=0 \\
\left(\bar{K}_{x y}\right)_{\text {noodle }}=0 \\
\left(\bar{D}_{x}\right)_{\text {noodle }}=\frac{4\left[\left(E_{1} I_{2}\right)_{\text {noodle }}+\widetilde{z}_{\text {noodle }}^{2}\left(E_{1} A\right)_{\text {noodle }}\right.}{l_{1 \& 4}+l_{2 \& 3}} \\
\left(\bar{D}_{y}\right)_{\text {noodle }}=0 \\
\left(\bar{D}_{x y}\right)_{\text {noodle }}=\frac{4\left(G_{23} J\right)_{\text {noodle }}}{l_{1 \& 4}+l_{2 \& 3}}
\end{gathered}
$$

where $\widetilde{z}_{\text {noodle }}$ is the offset of the noodle centroid, and $A, I_{2}$ and $J$ are the cross-section properties for the triangle representing the noodle region. Equations (A4) - (A9) are used to calculate the stiffness terms (for the fluted-core repeated unit shown in Fig. A1) that are substituted in Eq. (A2) to determine the global buckling load for the flutedcore cylinder.

\section{Acknowledgements}

This work was conducted as part of the NASA Engineering and Safety Center (NESC) Shell Buckling Knockdown Factor Project, NESC Assessment \#:07-010-E.

\section{References}

${ }^{1}$ Noyes, J. V., "Design of an Aircraft Utilizing Fiberglass Reinforced Plastic Primary Structure," Journal of Aircraft, Vol. 3, No. 5, Sept.-Oct., 1966, pp. 436-442.

${ }^{2}$ Bushnell, D., and Rankin, C., "Use of GENOPT and BIGBOSOR4 to Obtain Optimum Designs of an Axially Compressed Cylindrical Shell with a Composite Truss-Core Sandwich Wall", AIAA-2011-1811, 52 nd AIAA/ASME/ASCE/AHS/ASC Structures, Structural Dynamics and Materials Conference, Denver, Colorado, Apr. 4-7, 2011. 
${ }^{3}$ Schultz, M.R., Oremont, L., Guzman, J.C., McCarville, D., Rose, C.A., and Hilburger, M.W., "Compression Behavior of Fluted-Core Composite Panels," AIAA-2011-2170, 52 ${ }^{\text {nd }}$ AIAA/ASME/ASCE/AHS/ASC Structures, Structural Dynamics \& Materials Conference, Denver, CO, April 4-7, 2011.

${ }^{4}$ Oremont, L., and Schultz, M., "An Efficient Analysis Methodology for Fluted-Core Composite Structures," To be presented, 53 ${ }^{\text {rd }}$ AIAA/ASME/ASCE/AHS/ASC Structures, Structural Dynamics and Materials Conference, Honolulu, HI, April 23-26, 2012.

${ }^{5}$ Jegley, Dawn C., "A Study of the Structural Efficiency of Fluted Core Graphite-Epoxy Panels", NASA TM 101681, January 1990.

${ }^{6}$ Petersen, J. P., and Anderson, J. K., "Test of a Truss-core Sandwich Cylinder Loaded to Failure in Bending," NASA TN D3157, December, 1965.

${ }^{7}$ Robinson, M. J., Johnson, S. E., Eichinger, J. D., Hand, M. L., and Sorensen, E. T., "Trade Study Results for a SecondGeneration Reusable Launch Vehicle Composite Hydrogen Tank," AIAA 2004-1932, 45 ${ }^{\text {th }}$ AIAA/ASME/ASCE/AHS/ASC Structures, Structural Dynamics \& Materials Conference, Palm Springs, California, Apr. 19-22, 2004.

${ }^{8}$ Reeder, James R., "Property Values for Preliminary Design of the Ares I Composite Interstage,” Memo, 14 March 2007, pg. 7, Table $1,1^{\text {st }}$ column.

${ }^{9}$ Bushnell, D., "GENOPT-A Program that Writes User-Friendly Optimization Code", International Journal of Solids and Structures, Vol. 26, No. 9/10, pp. 1173-1210, 1990.

${ }^{10}$ Bushnell, D.: "BOSOR4-Program for Stress Stability and Vibration of Complex, Branched shells of Revolution", in Structural Analysis Systems, A. Niku-Lari, Editor, Vol. 2, pp. 25-54, Pergamon Press, 1986.

${ }^{11}$ Bushnell, David, "Automated Optimum Design of Shells of Revolution with Application to Ring-Stiffened Cylindrical Shells with Wavy Walls", AIAA-2000-1663, 41 ${ }^{\text {st }}$ AIAA/ASME/ASCE/AHS/ASC Structures, Structural Dynamics and Materials Conference, Atlanta, GA, April 3-6, 2000.

${ }^{12}$ Libove, C., and Hubka, R. E., "Elastic Constants for Corrugated-Core Sandwich Plates," NACA TN 2289, February, 1951.

${ }^{13}$ Seide, P., "The Stability Under Longitudinal Compression of Flat Symmetric Corrugated-Core Sandwich Plates with Simply Supported Loaded Edges and Simply Supported or Clamped Unloaded Edges," NACA TN 2679, April, 1952.

${ }^{14}$ Anderson, M. S., "Optimum Proportions for Truss-Core and Web-Core Sandwich Plates Loaded in Compression," NASA TN D-98, September, 1959.

${ }^{15}$ Lok, T. S., Cheng, Q., and Heng, L., "Equivalent Stiffness Parameters of Truss-Core Sandwich Panel," Proceedings of the Ninth International Offshore and Polar Engineering Conference, Brest, France, May 30 - June 4, 1999, pp. $292-298$.

${ }^{16}$ Lok, T. S., and Cheng, Q., "Elastic Stiffness Properties and Behavior of Truss-Core Sandwich Panel," Journal of Structural Engineering, Vol. 126, No. 5, May, 2000, pp. 552-559.

${ }^{17}$ Cheng, Q. H., Lee, H. P., and Lu, C., "A Numerical Analysis Approach for Evaluating Elastic Constants of Sandwich Structures with Various Cores," Composite Structures, Vol. 74, 2006, pp. 226-236.

${ }^{18}$ Romanoff, J., and Varsta, P., "Bending Response of Web-Core Sandwich Plates," Composite Structures, Vol. 81, 2007, pp. 292-302.

${ }^{19}$ Fung, T. C., Tan, K. H., and Lok, T. S., "Shear Stiffness $\mathrm{D}_{\mathrm{Qy}}$ for C-Core Sandwich Panels," Journal of Structural Engineering, Vol. 122, No. 8, August, 1996, pp. 958-966.

${ }^{20}$ Fung, T. C, and Tan, K. H., "Shear Stiffness for Z-Core Sandwich Panels," Journal of Structural Engineering, Vol. 124, No. 7, July, 1998, pp. 809-816.

${ }^{21}$ Zangani, D., Robinson, M., and Gibson, A. G., "Evaluation of Stiffness Terms for Z-Cored Sandwich Panels," Applied Composite Materials, Vol. 14, No. 3, May 2007, pp. 159-175.

${ }^{22}$ Bednarcyk, B. A., Yarrington, P. W., Lucking, R. C., Collier, C. S., and Ainsworth, J. J., "Efficient Design and Analysis of Lightweight Reinforced Core Sandwich and PRSEUS Structures," AIAA-2011-1909, 52 ${ }^{\text {nd }}$ AIAA/ASME/ASCE/AHS/ASC Structures, Structural Dynamics and Materials Conference, Denver, Co., April 4-7, 2011.

${ }^{23}$ Anonymous, "Buckling of Thin-walled Circular Cylinders", NASA SP-8007, September 1965 (Revised August 1968).

${ }^{24}$ Jones, R., "Buckling of Circular Cylindrical Shells with Multiple Orthotropic Layers and Eccentric Stiffeners", AIAA Journal, V. 6, No. 12, 1968, pp. 2301-2305.

${ }^{25}$ Vinson, J. R., and Sierakowski, R. L., The Behavior of Structures Composed of Composite Materials, Klewer Academic Publishers, Dordrecht, The Netherlands, 1987.

${ }^{26}$ Jones, R. M., Mechanics of Composite Materials, Hemisphere Publishing Corporation, New York, 1975.

${ }^{27}$ MSC/NASTRAN 2010, Software Package, MSC.Software Corporation, Santa Ana, CA, 2010.

${ }^{28}$ Abaqus/Standard, Software Package, Ver. 6.9.1, SIMULIA, Providence, RI, 2009.

${ }^{29}$ Rankin, C. C., Loden, W. A., Brogan, F. A., and Cabiness, H. D., "STAGS Users Manual," Rhombus Consultants Group, Inc., Ste. D201, 1121 San Antonio Rd., Palo Alto, CA 94303, January, 2007. 
Table 1: Summary of analysis results for fluted-core cylinder.

\begin{tabular}{|c|c|c|}
\hline Analysis Method & Mode & Load (kips) \\
\hline \multirow{3}{*}{ Analytical } & Global Buckling & 3,715 \\
\cline { 2 - 3 } & Local Buckling & 5,631 \\
\cline { 2 - 3 } & Material Failure & 3,035 \\
\hline \multirow{3}{*}{ GENOPT/BIGBOSOR4 } & 2 \\
& Global Buckling & 2,513 \\
\cline { 2 - 3 } & Local Buckling & 5,977 \\
\cline { 2 - 3 } & Material Failure & 2,536 \\
\hline \multirow{3}{*}{ Finite Element (NASTRAN) } & Global Buckling & 2,535 \\
\cline { 2 - 3 } & Local Buckling & 3,371 \\
\cline { 2 - 3 } & Material Failure & 3,004 \\
\hline
\end{tabular}

Table 2: Critical global buckling loads noodle-less fluted-core cylinder.

\begin{tabular}{|c|c|c|}
\hline \multirow{2}{*}{ Method } & \multicolumn{2}{|c|}{ Critical Global Buckling Load (kips) } \\
\hline Analytical Solution & \multicolumn{2}{|c|}{3,446} \\
\hline & Original & Refined \\
\hline NASTRAN & 2,142 & 2,098 \\
\hline Abaqus & 3,356 & 2,262 \\
\hline
\end{tabular}

Table 3: Axial transverse-shear displacements.

\begin{tabular}{|c|c|c|c|}
\cline { 2 - 4 } \multicolumn{1}{c|}{} & \multicolumn{3}{c|}{ Displacement (in.) } \\
\hline Mesh & NASTRAN & STAGS & Abaqus \\
\hline $1 / 1$ & $1.2181 \mathrm{E}-04$ & $1.2967 \mathrm{E}-04$ & $1.2146 \mathrm{E}-04$ \\
\hline $2 / 2$ & $1.3450 \mathrm{E}-04$ & $1.3734 \mathrm{E}-04$ & $1.3394 \mathrm{E}-04$ \\
\hline
\end{tabular}


Table 4: Circumferential transverse-shear displacements.

\begin{tabular}{|c|c|c|c|}
\cline { 2 - 4 } \multicolumn{1}{c|}{} & \multicolumn{3}{c|}{ Displacement (in.) } \\
\hline Mesh & NASTRAN & STAGS & Abaqus \\
\hline $1 / 1$ & $1.4917 \mathrm{E}-04$ & $1.5087 \mathrm{E}-04$ & $2.8111 \mathrm{E}-05$ \\
\hline $1 / 2$ & $1.4913 \mathrm{E}-04$ & N/A & $4.7438 \mathrm{E}-05$ \\
\hline $1 / 3$ & $1.4913 \mathrm{E}-04$ & N/A & $4.8072 \mathrm{E}-05$ \\
\hline $1 / 4$ & $1.4913 \mathrm{E}-04$ & N/A & $4.8259 \mathrm{E}-05$ \\
\hline $2 / 2$ & $1.4905 \mathrm{E}-04$ & $1.5364 \mathrm{E}-04$ & $1.2107 \mathrm{E}-04$ \\
\hline $2 / 4$ & $1.4905 \mathrm{E}-04$ & N/A & $1.2843 \mathrm{E}-04$ \\
\hline $3 / 4$ & $1.4907 \mathrm{E}-04$ & N/A & $1.3870 \mathrm{E}-04$ \\
\hline
\end{tabular}

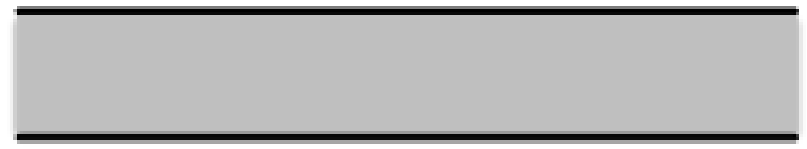

a) Traditional core (e.g., foam, honeycomb)

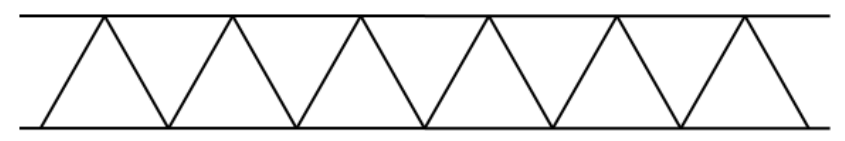

c) Triangle fluted core



e) Diamond fluted core

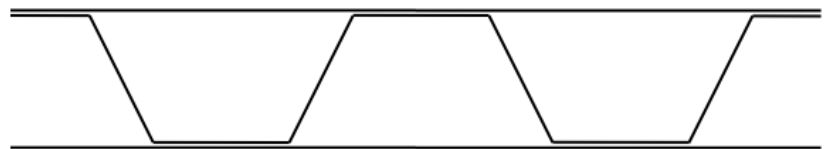

b) Corrugated core

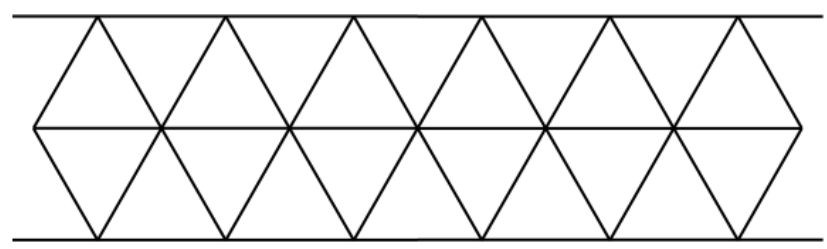

d) Two-layer triangle fluted core

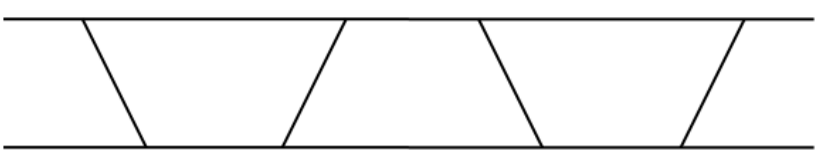

f) Trapezoid fluted core

Figure 1: Representative sandwich construction patterns.

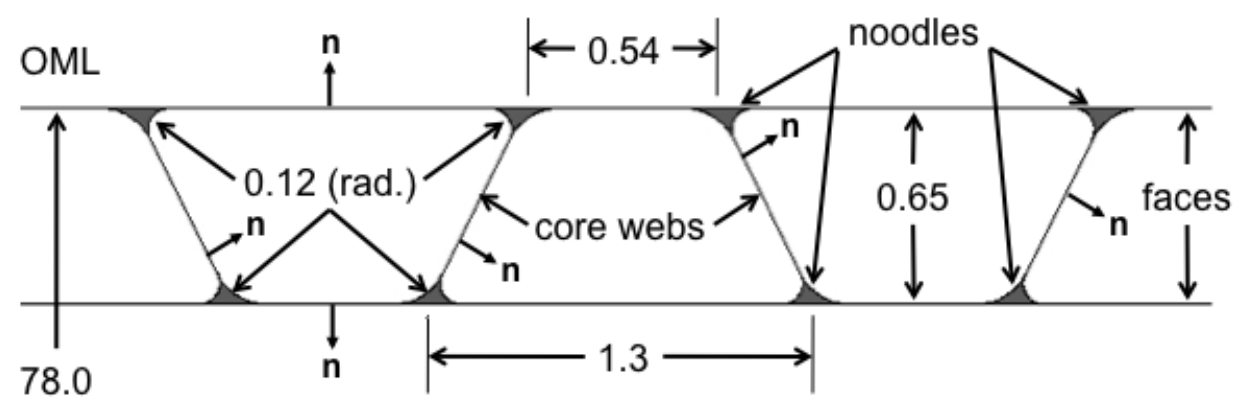

(cylinder OML radius)

Figure 2: Geometry of fluted-core cross section (in.). 


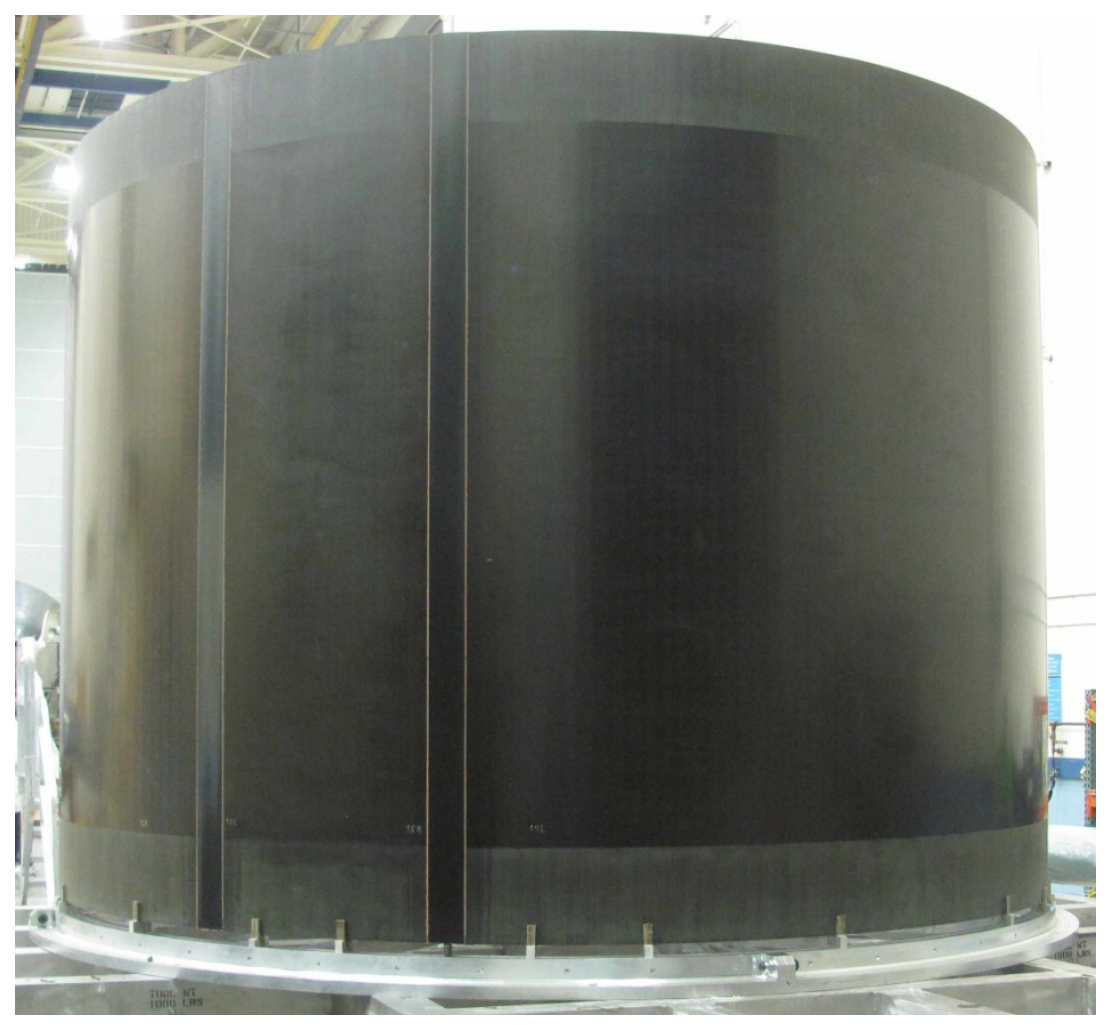

Figure 3: Fluted-core test cylinder for axial compression. (Photo courtesy of The Boeing Company)



Figure 4: Finite-element model of fluted-core cylinder for global buckling. 


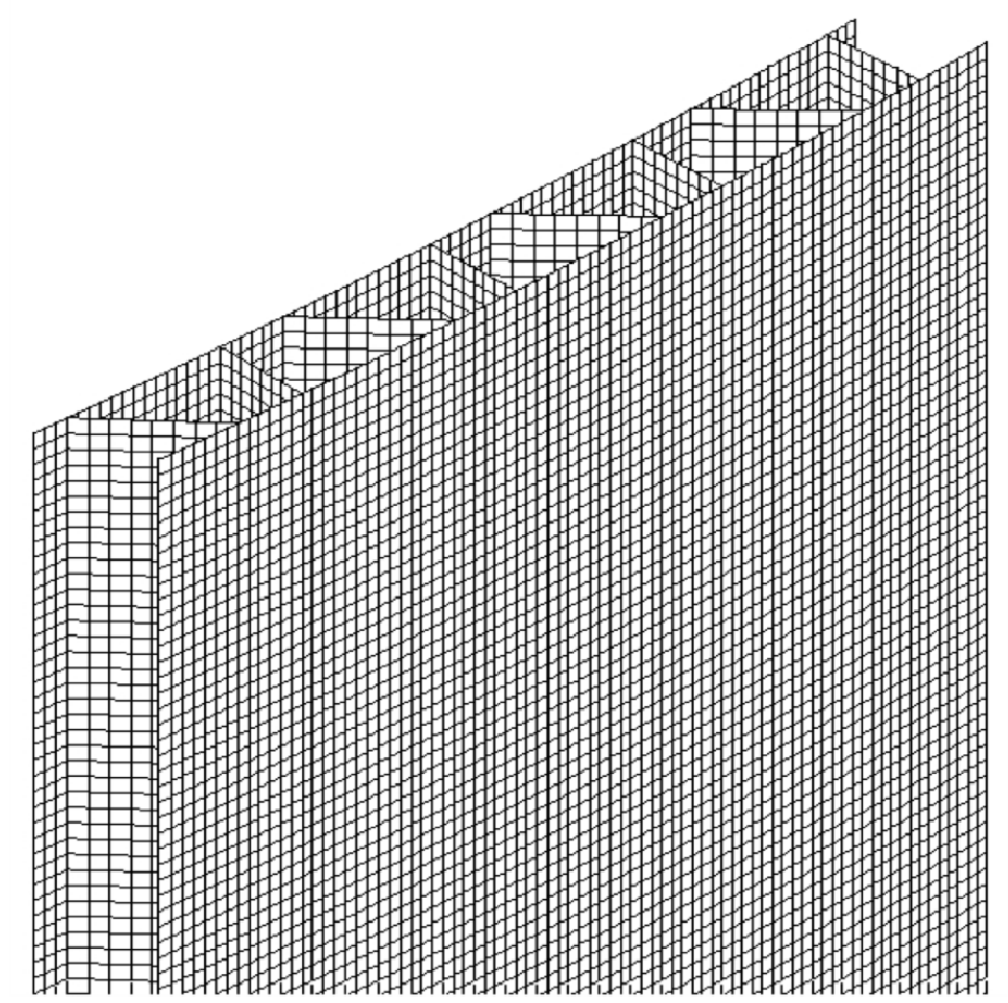

Figure 5: Close-up of the end of the local model used for local buckling and material failure predictions.

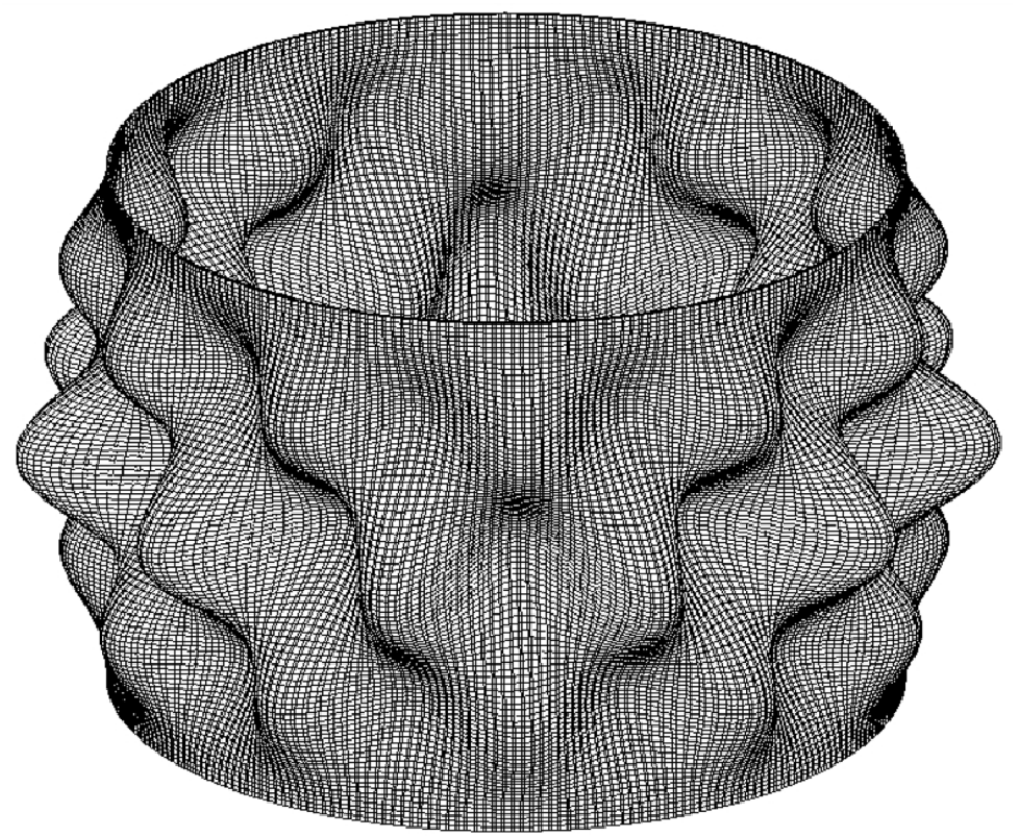

Figure 6: NASTRAN critical buckling mode, occurring at total load of 2,535 kips. 


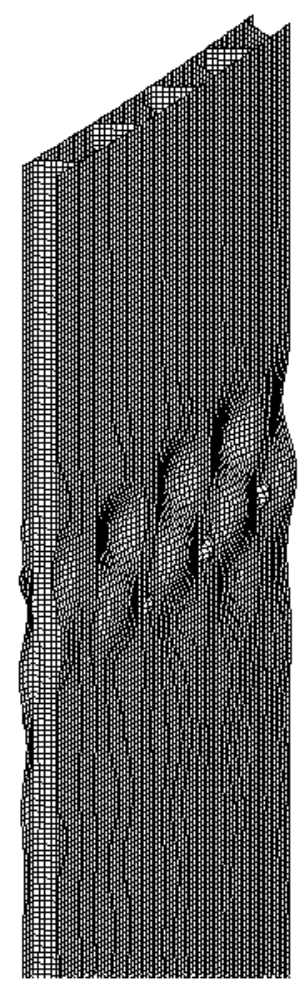

Figure 7: NASTRAN critical local buckling mode, occurring at total load of 3,371 kips.

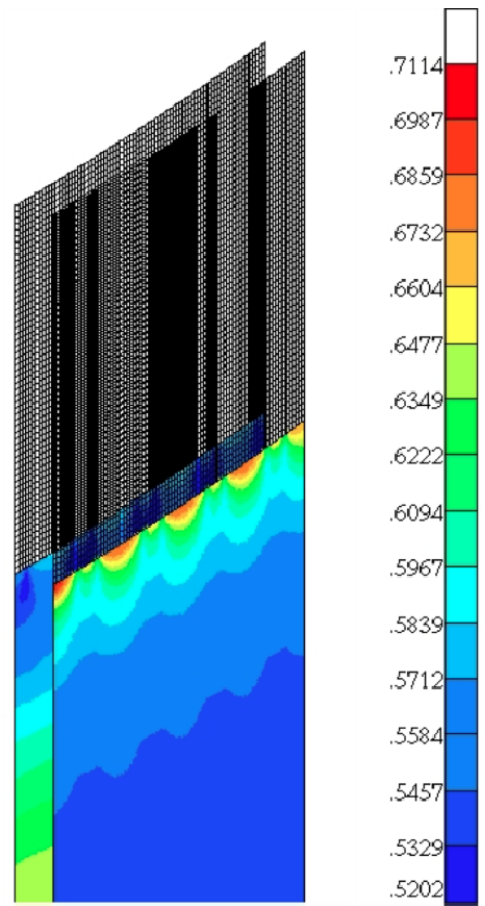

a) Faces (contours not shown in doubler region)

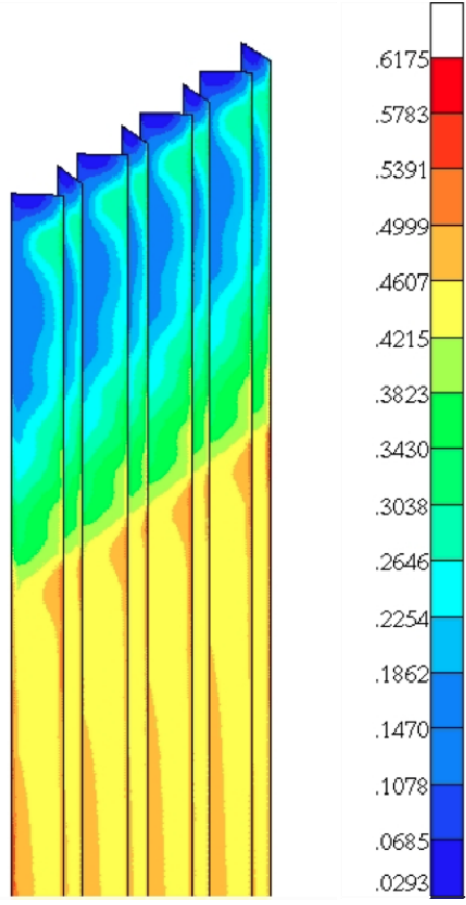

b) Webs

Figure 8: Maximum failure indices calculated using NASTRAN. 




a) NASTRAN

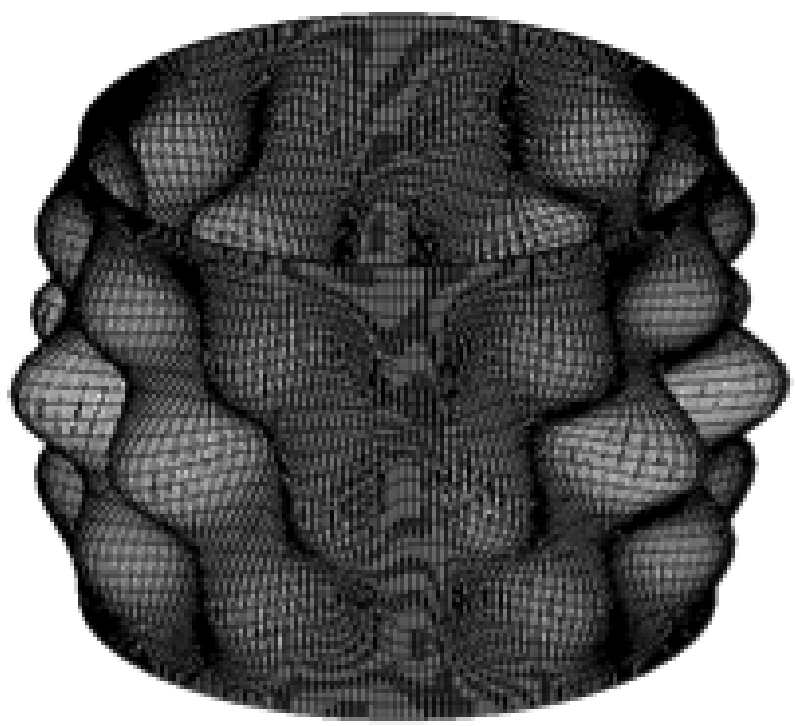

b) Abaqus

Figure 9: Critical buckling modes for noodle-less composite cylinder, one element per segment span. 


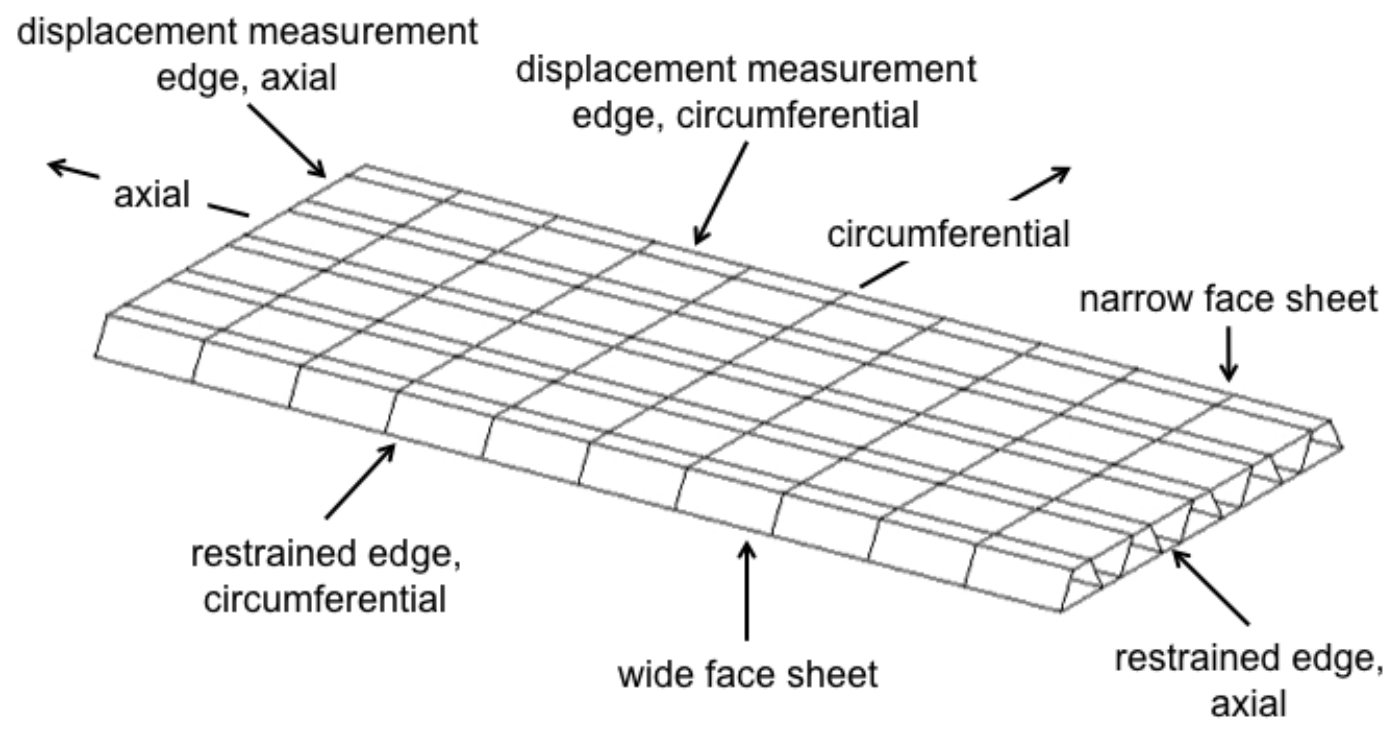

1/1 Mesh

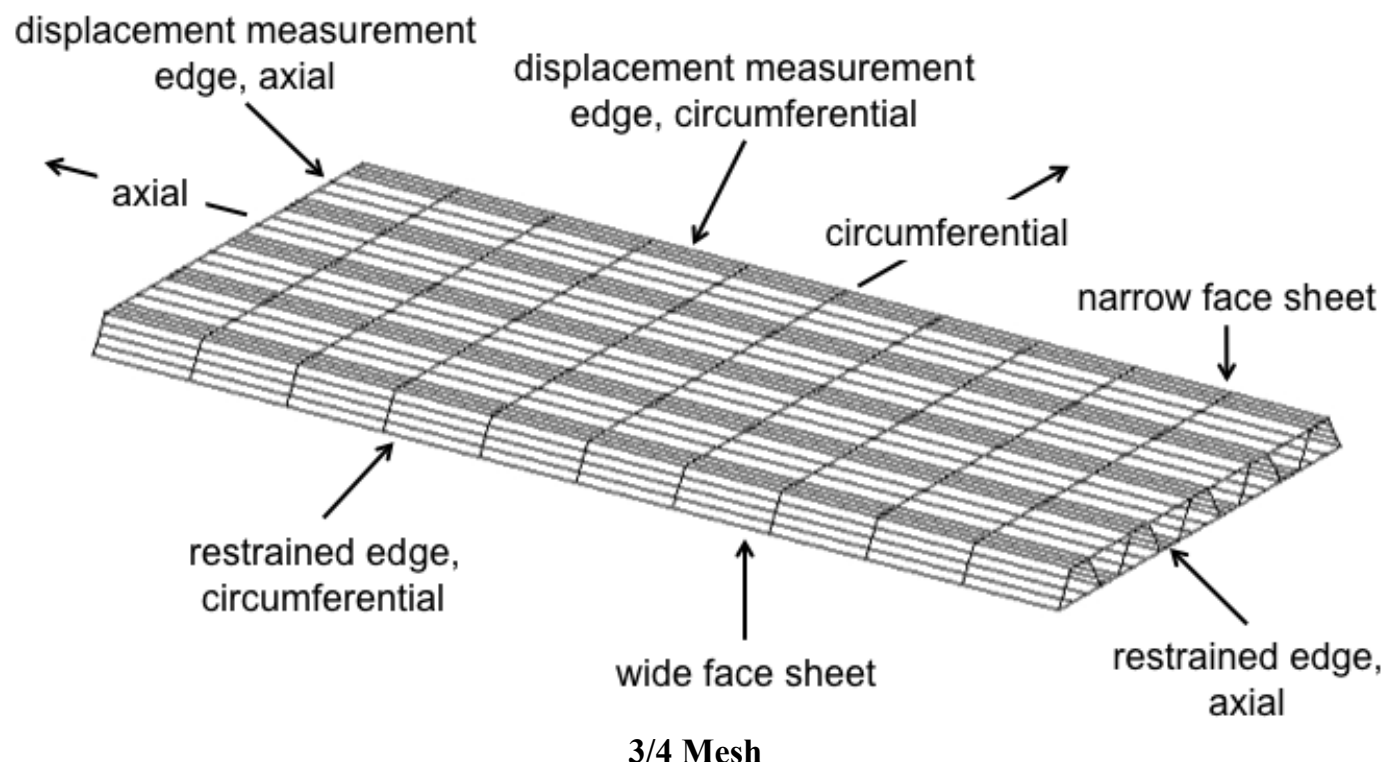

Figure 10: Representative meshes used for fluted-core transverse-shear study. Although the models are in the study are flat, axial and circumferential are used to relate the fluted-core to the cylinder. 




1/1 Mesh

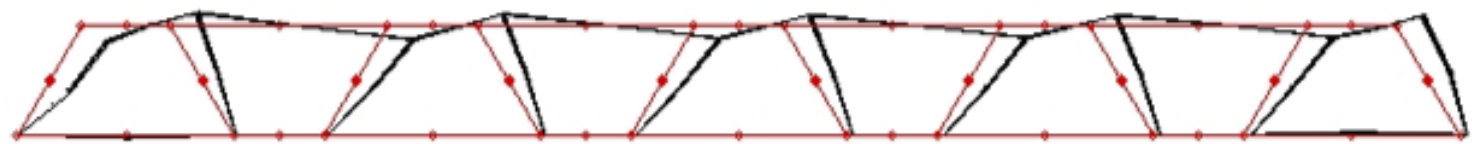

2/2 Mesh

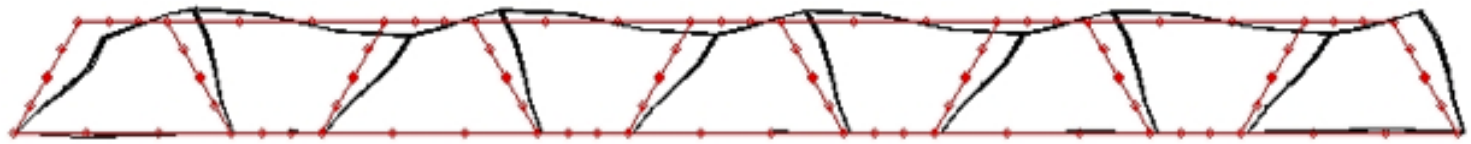

3/4 Mesh

Figure 11: NASTRAN shear deformation of fluted-core with loading in circumferential direction. (Deformations magnified by $\mathbf{1 0}^{3}$ )

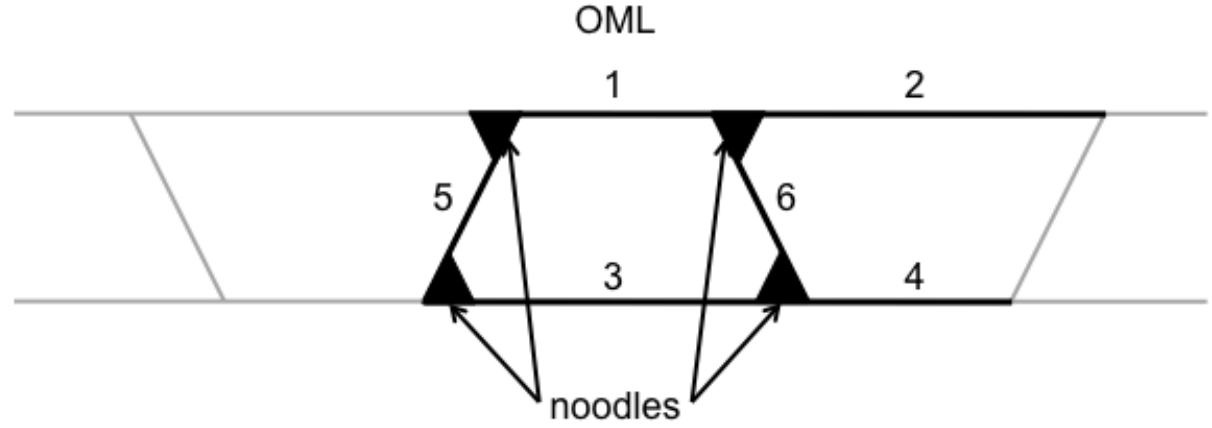

IML

Figure A1: Fluted-core repeated unit cell for analytical solution, showing the six segments and four noodles included in the calculations. 